\title{
Decision Matrix Equation and Block Diagram of Multilayer Electromagnetoelastic Actuator Micro and Nanodisplacement for Communications Systems
}

\author{
Sergey Mikhailovich Afonin \\ National Research University of Electronic Technology (MIET), Moscow, Russia; \\ eduems@mail.ru
}

\begin{abstract}
For the communications systems the parametric block diagram of the multilayer electromagnetoelastic actuator micro and nanodisplacement or the multilayer piezoactuator is determined in contrast to Cady and Mason's electrical equivalent circuits for the calculation of the piezoelectric transmitter and receiver, the vibration piezomotor. The decision matrix equation of the multilayer electromagnetoelastic actuator is used. The parametric block diagram of multilayer electromagnetoelastic actuator is obtained with the mechanical parameters the displacement and the force. The transfer functions of the multilayer electroelastic actuator are determined. The the generalized parametric block diagram, the generalized matrix equation for the multilayer electromagnetoelastic actuator micro and nanodisplacement are obtained. The deformations of the multilayer electroelastic actuator for the nanotechnology are described by the matrix equation. Block diagram and structural-parametric model of multilayer electromagnetoelastic actuator micro and nanodisplacement of the communications systems are obtained, its transfer functions are bult. Effects of geometric and physical parameters of multilayer electromagnetoelastic actuators and external load on its dynamic characteristics are determined. For calculations the communications systems with the multilayer piezoactuator for micro and nanodisplacement the parametric block diagram and the transfer functions of the multilayer piezoactuator are obtained.
\end{abstract}

Keywords: Multilayer electromagnetoelastic actuator; Parametric block diagram; Matrix transfer function; Multilayer piezoactuator.

\section{Introduction}

The multilayer electromagnetoelastic actuator is used for precise alignment in the range of movement from nanometers to tens of micrometers in nanotechnology, adaptive optics, communications systems. In the work the block diagram of multilayer electromagnetoelastic actuator for micro and nanodisplacement on the piezoelectric, piezomagnetic, electrostriction, magnetostriction effects, for example, the block diagram of the multilayer piezoactuator is determined in contrast to Cady and Mason's electrical equivalent circuits for the calculation of the piezotransmitter and piezoreceiver, the vibration piezomotor [1 - 11]. The block diagram of multilayer electromagnetoelastic actuator is obtained with the mechanical parameters the displacement and the force. Piezoactuator is piezomechanical device intended 
Sergey Mikhailovich Afonin; Decision Matrix Equation and Block Diagram of Multilayer Electromagnetoelastic Actuator Micro and Nanodisplacement for Communications Systems, Transactions on Networks and Communications, Volume 7 No. 3, June (2019); pp: 11-21

for actuation of mechanisms, systems or management based on the piezoeffect, converts the electrical signals into the mechanical movement or the force [11 - 15].

The investigation of the static and dynamic characteristics of the multilayer piezoactuator is necessary for the calculation mechatronics systems of the micro and nanometric movements for the communications systems. The multilayer piezoactuators are used in the micro and nanomanipulators for scanning tunneling microscopes and atomic force microscopes [7 - 23].

By decision matrix equation of the multilayer electromagnetoelastic actuator with allowance for the corresponding equation of the electromagnetoelasticity, the boundary conditions on loaded working surfaces of the multilayer electromagnetoelastic actuators, and the strains along the coordinate axes, it is possible to construct a structural parametric model of the multilayer electromagnetoelastic actuator [8, $9,16]$. The transfer functions and the parametric block diagrams of the multilayer electromagnetoelastic actuators are obtained from the set of equations describing the corresponding structural parametric model of the multilayer actuator for the communications systems. The solution of the matrix equation of the multilayer electromagnetoelastic actuator with the Laplace transform are used for the construction the parametric block diagram of the electromagnetoelastic actuator. As the result of the joint solution of the matrix equation of the multilayer actuator with the Laplace transform, the equation of the electromagnetoelasticity, the boundary conditions on the two loaded working surfaces of the multilayer actuator, we obtain the corresponding structural-parametric model and the parametric block diagram of the multilayer electromagnetoelastic actuator.

\section{Block diagram of multilayer electromagnetoelastic actuator}

For the communications systems the parametric block diagram and the matrix transfer functions of the multilayer electromagnetoelastic actuator are obtained from the structural-parametric model of the multilayer actuator with the mechanical parameters the displacement and the force. The parametric block diagrams of the voltage or current-controlled multilayer piezoactuator are determined using the equation of the inverse piezoeffect in the form $[8,11]$ :

for the voltage control

$$
S_{i}=d_{m i} E_{m}+S_{i j}^{E} T_{j}
$$

for the current control

$$
S_{i}=g_{m i} D_{m}+s_{i j}^{D} T_{j}
$$

where the indexes $i=1,2, \ldots, 6, j=1,2, \ldots, 6, m=1,2,3$, with $1,2,3$ are mutually perpendicular coordinate axes, $S_{i}$ is relative deformation with index $I, d_{m i}$ is the piezoelectric module, $E_{m}$ is electric field strength and $D_{m}$ is the electric induction along the axis $m, g_{m i}$ is the piezoelectric constant; $s_{i j}^{E}, s_{i j}^{D}$ are the elastic compliances with $E=$ const and $D=$ const, $T_{j}$ is the mechanical stress with index $j$, therefore we obtain $\Psi=E, D$ generalized control parameter in the form of the electric field strength or the electric induction for the voltage or current-controlled of the multilayer piezoactuator.

The equation of the direct piezoeffect has the form $[8,11]$ 


$$
D_{m}=d_{m i} T_{i}+\varepsilon_{m k}^{T} E_{k}
$$

where the indexes $m=1,2,3, k=1,2,3, \varepsilon_{m k}^{T}$ is the dielectric constants for $T=$ const .

Accordingly in general the equation of the electromagnetoelasticity of the multilayer electromagnetoelastic actuator $[11,14,16,20]$ has the form

$$
S_{i}=v_{m i} \Psi_{m}(t)+s_{i j}^{\Psi} T_{j}(x, t)
$$

where $S_{i}=\partial \xi(x, t) / \partial x$ is the relative displacement along axis $i$ of the cross section of the actuator, therefore we obtain $\Psi=E, D, H$ generalized control parameter in the form $E_{m}$ for the voltage control, $D_{m}$ for the current control, $H_{m}$ for magnetic field strength control along axis $m, T_{j}$ is the mechanical stress along axis $j, v_{m i}$ is the coefficient of electromagnetoelasticity, for example, piezoelectric module or magnetostriction coefficient, $s_{i j}^{\Psi}$ is the elastic compliance with $\Psi=$ const . The multilayer piezoactuator on Figure 1 consist from the piezolayers or the piezoplates connected electrically in parallel and mechanically in series.

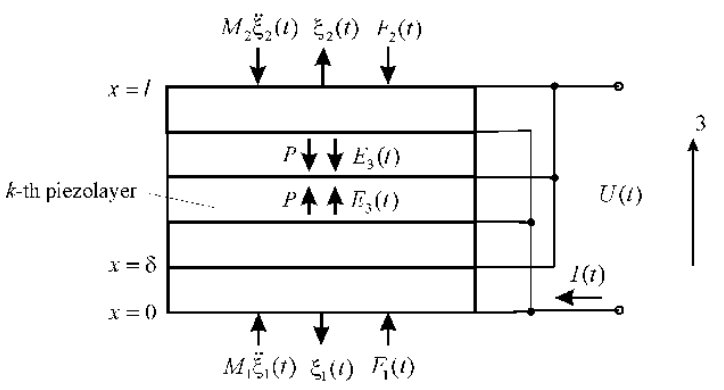

a)

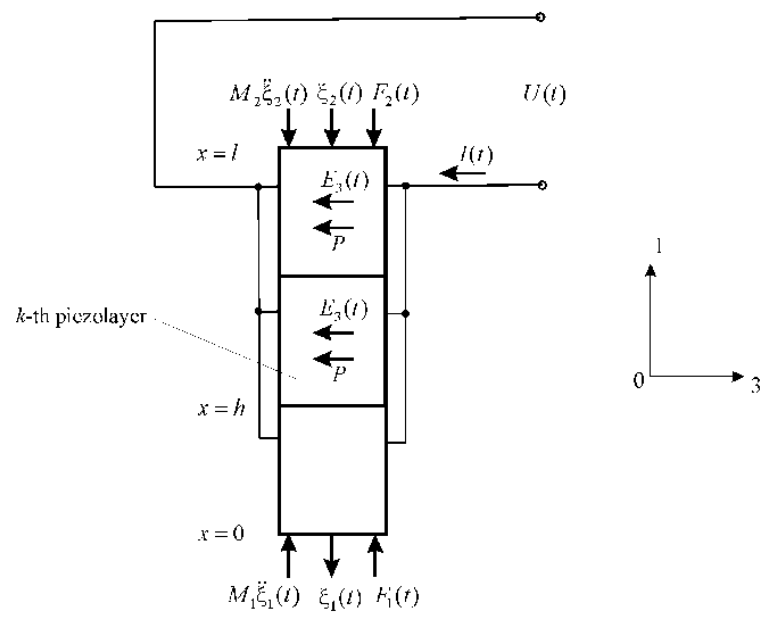

b)

Figure 1. The multilayer piezoactuator a) for the longitudinal piezoeffect, b) for the transverse piezoeffect

For example, we consider the matrix equation for the Laplace transforms of the forces and of the displacements [7] at the input and output ends of the $k$-th piezolayer of the multilayer piezoactuator from $n$ the layers. The equivalent $T$-shaped quadripole of the $k$-th piezolayer is shown on Figure 2. 


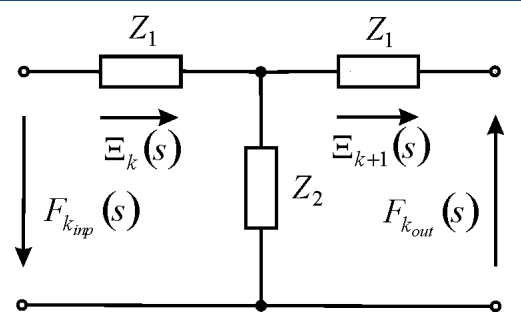

Figure 2. The quadripole for $k$-th piezolayer

The circuit of the multilayer piezoactuator on Fugure 3 is compiled from the equivalent $T$-shaped quadripole for the $k$-th piezolayer and the forces equations, acting on the faces the piezolayer. Therefore on Fugure 2 and Fugure 3 we have the Laplace transforms of the corresponding forces on the input and output faces of the $k$-th piezolayer of the multilayer piezoactuator in the form of the system of the equations for the equivalent $T$-shaped quadripole

$$
\begin{aligned}
& F_{k_{\text {inp }}}(s)=-\left(Z_{1}+Z_{2}\right) \Xi_{k}(s)+Z_{2} \Xi_{k+1}(s) \\
& -F_{k_{\text {out }}}(s)=-Z_{2} \Xi_{k}(s)+\left(Z_{1}+Z_{2}\right) \Xi_{k+1}(s)
\end{aligned}
$$

where $Z_{1}=\frac{S_{0} \gamma \operatorname{th}(\delta \gamma)}{S_{i j}^{\Psi}}, Z_{2}=\frac{S_{0} \gamma}{S_{i j}^{\Psi} \operatorname{sh}(\delta \gamma)}$ are the resistance of the equivalent quadripole of the $k$-th piezolayer, $\delta$ is the thickness on Figure $1 a, \gamma=\frac{s}{c^{\Psi}}+\alpha$ is the coefficient of wave propagation, $F_{k_{\text {inp }}}(s), F_{k_{\text {out }}}(s)$ are the Laplace transform of the forces at the input and output ends of the $k$-th piezolayer, $\Xi_{k}(s), \Xi_{k+1}(s)$ are the Laplace transforms of the displacements at input and output ends of the $k$-th piezolayer, $s$ is the Laplace operator, $c^{\Psi}$ is the speed of sound in the piezoceramics with $\Psi=$ const, $\alpha$ is the attenuation coefficient, $s_{i j}^{\Psi}$ is the elastic compliance with $\Psi=$ const .

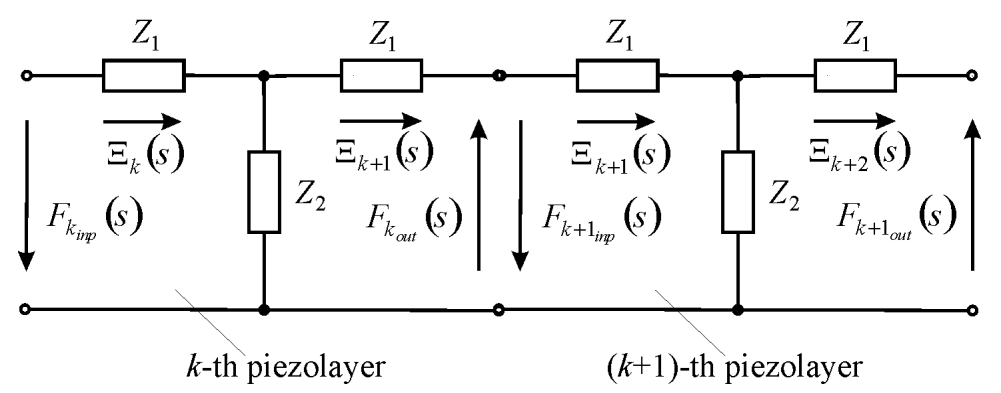

Figure 3. The circuit of the multilayer piezoactuator with the quadripoles for $k$-th and $(k+1)$-th piezolayers Accordingly we have for Figure 2 the Laplace transforms the following system of the equations for the $k$ th piezolayer in the form 


$$
\begin{gathered}
-F_{k_{\text {inp }}}(s)=\left(1+\frac{Z_{1}}{Z_{2}}\right) F_{k_{\text {out }}}(s)+Z_{1}\left(2+\frac{Z_{1}}{Z_{2}}\right) \Xi_{k+1}(s) \\
\Xi_{k}(s)=\frac{1}{Z_{1}} F_{k_{\text {out }}}(s)+\left(1+\frac{Z_{1}}{Z_{2}}\right) \Xi_{k+1}(s)
\end{gathered}
$$

the matrix equation for the $k$-th piezolayer

$$
\left[\begin{array}{c}
-F_{k_{\text {inp }}}(s) \\
\Xi_{k}(s)
\end{array}\right]=[M]\left[\begin{array}{c}
F_{k_{\text {out }}}(s) \\
\Xi_{k+1}(s)
\end{array}\right]
$$

and the matrix $[M]$ in the form

$$
[M]=\left[\begin{array}{ll}
m_{11} & m_{12} \\
m_{21} & m_{22}
\end{array}\right]=\left[\begin{array}{cc}
1+\frac{Z_{1}}{Z_{2}} & Z_{1}\left(2+\frac{Z_{1}}{Z_{1}}\right) \\
\frac{1}{Z_{2}} & 1+\frac{Z_{1}}{Z_{2}}
\end{array}\right]
$$

where $m_{11}=m_{22}=1+\frac{Z_{1}}{Z_{2}}=\operatorname{ch}(\delta \gamma), m_{12}=Z_{1}\left(2+\frac{Z_{1}}{Z_{1}}\right)=Z_{0} \operatorname{sh}(\delta \gamma), m_{21}=\frac{1}{Z_{2}}=\frac{\operatorname{sh}(\delta \gamma)}{Z_{0}}, Z_{0}=\frac{S_{0} \gamma}{s_{i j}^{\Psi}}$.

For the multilayer piezoactuator of the Laplace transform the displacement $\Xi_{k+1}(s)$ and the force $F_{k_{\text {out }}}(s)$ acting on the output face of the $k$-th layer on Figure 3 are corresponded to Laplace transforms of displacement and force acting on the input face of the $(k+1)$-th layer.

The force on the output face for the $k$-th piezolayer is equal in magnitude and opposite in direction to the force on the input face for the $(k+1)$-th piezolayer, hence

$$
F_{k_{\text {out }}}(s)=-F_{k+\text { linp }_{1}}(s)
$$

From equation (7) the matrix equation for $n$ piezolayers of the multilayer piezoactuator has the form

$$
\left[\begin{array}{c}
-F_{1_{\text {inp }}}(s) \\
\Xi_{1}(s)
\end{array}\right]=[M]^{n}\left[\begin{array}{c}
F_{n_{\text {out }}}(s) \\
\Xi_{n+1}(s)
\end{array}\right]
$$

with the matrix of the multilayer piezoactuator

$$
[M]^{n}=\left[\begin{array}{cc}
\operatorname{ch}(n \delta \gamma) & Z_{0} \operatorname{sh}(n \delta \gamma) \\
\frac{\operatorname{sh}(n \delta \gamma)}{Z_{0}} & \operatorname{ch}(n \delta \gamma)
\end{array}\right]
$$

Accordingly in general the matrix for the equivalent quadripole of the multilayer electromagnetoelastic actuator has the form

$$
[M]^{n}=\left[\begin{array}{cc}
\operatorname{ch}(l \gamma) & Z_{0} \operatorname{sh}(l \gamma) \\
\frac{\operatorname{sh}(l \gamma)}{Z_{0}} & \operatorname{ch}(l \gamma)
\end{array}\right]
$$


Sergey Mikhailovich Afonin; Decision Matrix Equation and Block Diagram of Multilayer Electromagnetoelastic Actuator Micro and Nanodisplacement for Communications Systems, Transactions on Networks and Communications, Volume 7 No. 3, June (2019); pp: 11-21

Therefore we have from the equation (9) the equivalent quadripole of the multilayer piezoactuator on Figure 1 for the longitudinal piezoeffect with length of the multilayer piezoactuator $l=n \delta$, for the transverse piezoeffect with length $l=n h$, for the shift piezoeffect with length $l=n b$, where $\delta, h, b$ are the thickness, the height, the width for the $k$-th piezolayer.

Equations of the forces acting on the faces of the multilayer piezoactuator:

$$
\begin{aligned}
& \text { at } x=0, T_{j}(0, s) S_{0}=F_{1}(s)+M_{1} p^{2} \Xi_{1}(s) \\
& \text { at } x=l, T_{j}(l, s) S_{0}=-F_{2}(s)-M_{2} p^{2} \Xi_{2}(s)
\end{aligned}
$$

where $T_{j}(0, s), T_{j}(l, s)$ are the Laplace transforms of mechanical stresses at the two ends of the multilayer piezoactuator.

The Laplace transform of the displacement and the force for the first face of the multilayer piezoactuator has the form

$$
\text { at } x=0 \text { and } \Xi_{1}(s), F_{1}(s)
$$

the Laplace transforms of the displacement and the forces for the the second face of the piezoactuator has the following form

$$
\text { at } x=l \text { and } \Xi_{2}(s)=\Xi_{n+1}(s), F_{2}(s)=F_{n_{\text {out }}}(s)
$$

Let us construct the structural-parametric model of the multilayer electromagnetoelastic actuator. From equation (4) the Laplace transform of the force, which causes the deformation, has the form

$$
F(s)=\frac{v_{m i} S_{0} \Psi_{m}(s)}{s_{i j}^{\Psi}}
$$

Accordingly we have the equations for the structural-parametric model and the generalized block diagram of the multilayer electromagnetoelastic actuator on Figure 4. The structural-parametric model is obtained in result analysis of the equation of the force that causes deformation, of the system of the equations for the equivalent quadripole of the multilayer electromagnetoelastic actuator, the equation of the forces on its faces in the following form

$$
\begin{aligned}
& \Xi_{1}(s)=\left[\frac{1}{M_{1} s^{2}}\right]\left\{-F_{1}(s)+\left(\frac{1}{\chi_{i j}^{\Psi}}\right)\left[\begin{array}{c}
\left.v_{m i} \Psi_{m}(s)-\left[\frac{\gamma}{\operatorname{sh}(l \gamma)}\right] \times\right] \\
\times\left[\operatorname{ch}(l \gamma) \Xi_{1}(s)-\Xi_{2}(s)\right]
\end{array}\right]\right\} \\
& \Xi_{2}(s)=\left[\frac{1}{M_{2} s^{2}}\right]\left\{-F_{2}(s)+\left(\frac{1}{\chi_{i j}^{\Psi}}\right)\left[\begin{array}{l}
v_{m i} \Psi_{m}(s)-\left[\frac{\gamma}{\operatorname{sh}(l \gamma)}\right] \times \\
\times\left[\operatorname{ch}(l \gamma) \Xi_{2}(s)-\Xi_{1}(s)\right]
\end{array}\right]\right\}
\end{aligned}
$$


where $v_{m i}=\left\{\begin{array}{l}d_{33}, d_{31}, d_{15} \\ g_{33}, g_{31}, g_{15} \\ d_{33}, d_{31}, d_{15}\end{array}, \Psi_{m}=\left\{\begin{array}{l}E_{3}, E_{1} \\ D_{3}, D_{1} \\ H_{3}, H_{1}\end{array}, s_{i j}^{\Psi}=\left\{\begin{array}{l}s_{33}^{E}, s_{11}^{E}, s_{55}^{E} \\ s_{33}^{D}, s_{11}^{D}, s_{55}^{D}, c^{\Psi} \\ s_{33}^{H}, s_{11}^{H}, s_{55}^{H}\end{array}=\left\{\begin{array}{l}c^{E} \\ c^{D} \\ c^{H}\end{array}, \gamma=\left\{\begin{array}{l}\gamma^{E} \\ \gamma^{D} \\ \gamma^{H}\end{array}, l=\left\{\begin{array}{l}\delta \\ h, \chi_{i j}^{\Psi}=s_{i j}^{\Psi} \\ b\end{array}\right.\right.\right.\right.\right.\right.$

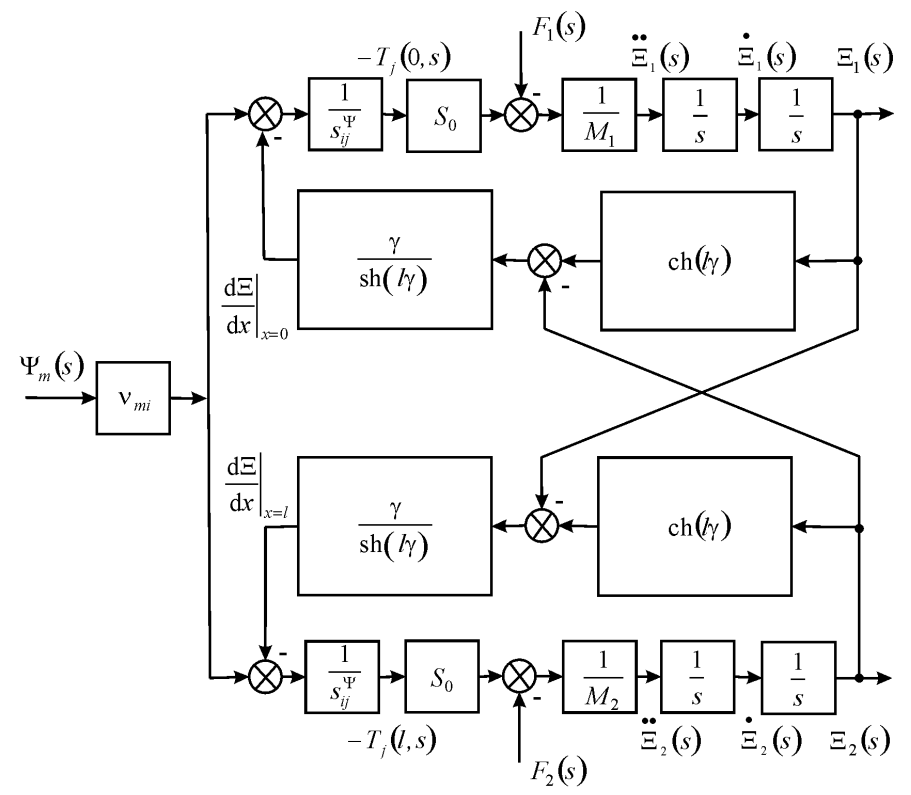

Figure 4. The generalized block diagram of the electromagnetoelastic actuator

The generalized block diagram of the electromagnetoelastic actuator is constructed using the generalized structural-parametric model of the multilayer electromagnetoelastic actuator micro and nanodisplacement for the communications systems.

\section{Matrix transfer function of multilayer electromagnetoelastic actuator}

The matrix transfer function of the multilayer electromagnetoelastic actuator determined from its structural-parametric model (15) has the form

$$
\left[\begin{array}{l}
\Xi_{1}(s) \\
\Xi_{2}(s)
\end{array}\right]=\left[\begin{array}{lll}
W_{11}(s) & W_{12}(s) & W_{13}(s) \\
W_{21}(s) & W_{22}(s) & W_{23}(s)
\end{array}\right]\left[\begin{array}{l}
\Psi_{m}(s) \\
F_{1}(s) \\
F_{2}(s)
\end{array}\right]
$$

Therefore in general the matrix transfer function of the multilayer electromagnetoelastic actuator is obtained in following form

$$
\begin{gathered}
{[\Xi(s)]=[W(s)][P(s)]} \\
{[\Xi(s)]=\left[\begin{array}{l}
\Xi_{1}(s) \\
\Xi_{2}(s)
\end{array}\right],[W(s)]=\left[\begin{array}{lll}
W_{11}(s) & W_{12}(s) & W_{13}(s) \\
W_{21}(s) & W_{22}(s) & W_{23}(s)
\end{array}\right],[P(s)]=\left[\begin{array}{c}
\Psi_{m}(s) \\
F_{1}(s) \\
F_{2}(s)
\end{array}\right]}
\end{gathered}
$$


Sergey Mikhailovich Afonin; Decision Matrix Equation and Block Diagram of Multilayer Electromagnetoelastic Actuator Micro and Nanodisplacement for Communications Systems, Transactions on Networks and Communications, Volume 7 No. 3, June (2019); pp: 11-21

where $[\Xi(s)]$ is the column-matrix of the Laplace transforms of the displacements, $[W(s)]$ is the matrix transfer function, $[P(s)]$ the column-matrix of the Laplace transforms of the control parameter and the forces.

The generalized transfer functions of the electromagnetoelastic actuator are the ratio of the Laplace transform of the displacement of the face and the Laplace transform of the corresponding control parameter or the force at zero initial conditions

$$
\begin{gathered}
W_{11}(s)=\Xi_{1}(s) / \Psi_{m}(s)=v_{m i}\left[M_{2} \chi_{i j}^{\Psi} s^{2}+\gamma \operatorname{th}(l \gamma / 2)\right] / A_{i j} \\
\chi_{i j}^{\Psi}=s_{i j}^{\Psi} / s_{0} \\
A_{i j}=M_{1} M_{2}\left(\chi_{i j}^{\Psi}\right)^{2} s^{4}+\left\{\left(M_{1}+M_{2}\right) \chi_{i j}^{\Psi} /\left[c^{\Psi} \operatorname{th}(l \gamma)\right] s^{3}+\right. \\
+\left[\left(M_{1}+M_{2}\right) \chi_{i j}^{\Psi} \alpha / \operatorname{th}(l \gamma)+1 /\left(c^{\Psi}\right)^{2} s^{2}+2 \alpha s / c^{\Psi}+\alpha^{2}\right. \\
W_{21}(s)=\Xi_{2}(s) / \Psi_{m}(s)=v_{i j}\left[M_{1} \chi_{i j}^{\Psi} s^{2}+\gamma \operatorname{th}(l \gamma / 2)\right] / A_{i j} \\
\begin{array}{c}
W_{12}(s)=\Xi_{1}(s) / F_{1}(s)=-\chi_{i j}^{\Psi}\left[M_{2} \chi_{i j}^{\Psi} s^{2}+\gamma / \operatorname{th}(l \gamma)\right] / A_{i j} \\
W_{13}(s)=\Xi_{1}(s) / F_{2}(s)= \\
=W_{22}(s)=\Xi_{2}(s) / F_{1}(s)=\left[\chi_{i j}^{\Psi} \gamma / \operatorname{sh}(l \gamma)\right] / A_{i j} \\
W_{23}(s)=\Xi_{2}(s) / F_{2}(s)=-\chi_{i j}^{\Psi}\left[M_{1} \chi_{i j}^{\Psi} s^{2}+\gamma / \operatorname{th}(l \gamma)\right] / A_{i j}
\end{array}
\end{gathered}
$$

From generalized structural-parametric model of the multilayer electromagnetoelastic actuator its generalized block diagram and generalized matrix transfer function are determined to calculate the characteristics of the multilayer electromagnetoelastic actuator micro and nanodisplacement for the communications system.

For the approximation of the hyperbolic cotangent by two terms of the power series in the transfer function of the multilayer piezoactuator (16) for the elastic-inertial load with one fixed face at $M_{1} \rightarrow \infty$, $m<<M_{2}$ and for the longitudinal piezoeffect with the voltage control of the multilayer piezoactuator its block diagram on Figure 5 and expression of the transfer function are obtained in following form

$$
\begin{gathered}
W(s)=\frac{\Xi_{2}(s)}{U(s)}=\frac{d_{33} n}{\left(1+C_{e} / C_{33}^{E}\right)\left(T_{t}^{2} s^{2}+2 T_{t} \xi_{t} s+1\right)} \\
T_{t}=\sqrt{M_{2} /\left(C_{e}+C_{33}^{E}\right)}, \quad \xi_{t}=\alpha l^{2} C_{33}^{E} /\left[3 c^{E} \sqrt{M_{2}\left(C_{e}+C_{33}^{E}\right)}\right]
\end{gathered}
$$


where $\Xi_{2}(s), U(s)$ are the Laplace transforms the displacement face of the multilayer piezoactuator and the voltage, $T_{t}$ is the time constant and $\xi_{t}$ is the damping coefficient of the multilayer piezoactuator, $C_{33}^{E}=S_{0} /\left(S_{33}^{E} l\right)$ is the is rigidity of the multilayer piezoactuator for the longitudinal piezoeffect for $E=$ const

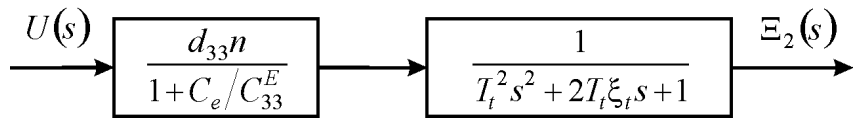

Figure 5. The block diagram of the voltage-controlled multilayer piezoactuator for the longitudinal piezoeffect and elastic-inertial load

Let us construct from (18) the transient response of the multilayer piezoactuator for the longitudinal piezoelectric effect with the voltage control. The expression for the transient response of the voltagecontrolled the multilayer piezoactuator for the longitudinal piezoeffect and elastic-inertial load is determined in the form

$$
\begin{gathered}
\xi(t)=\xi_{m}\left[1-\frac{e^{-\frac{\xi_{t} t}{T_{t}}}}{\sqrt{1-\xi_{t}^{2}}} \sin \left(\omega_{t} t+\varphi_{t}\right)\right] \\
\xi_{m}=\frac{d_{33} n U_{m}}{1+C_{e} / C_{33}^{E}}, \quad \omega_{t}=\sqrt{1-\xi_{t}^{2}} / T_{t}, \quad \varphi_{t}=\operatorname{arctg}\left(\sqrt{1-\xi_{t}^{2}} / \xi_{t}\right)
\end{gathered}
$$

where $\xi_{m}$ is the steady-state value of displacement of the multilayer piezoactuator and $U_{m}$ is the amplitude of the voltage.

For the voltage-controlled multilayer piezoactuator from the piezoceramics type of the lead zirconate titanate PZT under the longitudinal piezoelectric effect for the elastic-inertial load with one fixed face at $M_{1} \rightarrow \infty, m<<M_{2}$, the amplitude of the voltage $U_{m}=50 \mathrm{~V}, d_{33}=4 \cdot 10^{-10} \mathrm{~m} / \mathrm{V}, n=10, M=1 \mathrm{~kg}, C_{33}^{E}=$ $6 \cdot 10^{7} \mathrm{~N} / \mathrm{m}, C_{e}=0.4 \cdot 10^{7} \mathrm{~N} / \mathrm{m}$, values the steady-state value of displacement $\xi_{m}=200 \mathrm{~nm}$ and the time constant $T_{t}=0.125 \cdot 10^{-3} \mathrm{~s}$ are obtained.

\section{Conclusion}

The generalized parametric block diagram and the generalized structural-parametric model of the multilayer electromagnetoelastic actuator micro and nanodisplacement are constructed with the mechanical parameters the displacement and the force.

The parametric block diagrams of the multilayer piezoactuator for the transverse, longitudinal, shift piezoelectric effects are determined. The matrix transfer function of the multilayer electromagnetoelastic actuator is determined for the communications systems.

From the equation of the electromagnetoelasticity, the equation of the force that causes the deformation, the system of the equations for the equivalent quadripole of the multilayer actuator, the equations of the 
Sergey Mikhailovich Afonin; Decision Matrix Equation and Block Diagram of Multilayer Electromagnetoelastic Actuator Micro and Nanodisplacement for Communications Systems, Transactions on Networks and Communications, Volume 7 No.

3, June (2019); pp: 11-21

forces on its faces we obtain the structural-parametric model of the multilayer electromagnetoelastic actuator micro and nanodisplacement.

The parametric structural schematic diagram and the transfer functions of the multilayer piezoactuator are considered using the structural-parametric model of the piezoactuator for the communications systems.

\section{REFERENCES}

[1]. Schultz, J., Ueda, J., Asada, H., Cellular actuators. Oxford: Butterworth-Heinemann Publisher, 2017. 382 p.

[2]. Afonin, S.M., Absolute stability conditions for a system controlling the deformation of an elecromagnetoelastic transduser. Doklady mathematics, 2006. 74(3): p. 943-948, doi:10.1134/S1064562406060391.

[3]. Zhou, S., Yao, Z., Design and optimization of a modal-independent linear ultrasonic motor. IEEE transaction on ultrasonics, ferroelectrics, and frequency control, 2014. 61(3): p. 535-546, doi:10.1109/TUFFC.2014.2937.

[4]. Przybylski, J., Static and dynamic analysis of a flextensional transducer with an axial piezoelectric actuation. Engineering structures, 2015. 84: p. 140-151, doi:10.1016/j.engstruct.2014.11.025.

[5]. Ueda, J., Secord, T., Asada, H.H., Large effective-strain piezoelectric actuators using nested cellular architecture with exponential strain amplification mechanisms. IEEE/ASME transactions on mechatronics, 2010. 15(5): p. 770-782, doi:10.1109/TMECH.2009.2034973.

[6]. Karpelson, M., Wei, G.-Y., Wood, R.J., Driving high voltage piezoelectric actuators in microrobotic applications. Sensors and actuators A: Physical, 2012. 176: p. 78-89, doi:10.1016/j.sna.2011.11.035.

[7]. Afonin, S.M., Block diagrams of a multilayer piezoelectric motor for nano- and microdisplacements based on the transverse piezoeffect. Journal of computer and systems sciences international, 2015. 54(3): p. 424439, doi:10.1134/S1064230715020021.

[8]. Afonin, S.M., Structural parametric model of a piezoelectric nanodisplacement transduser. Doklady physics, 2008. 53(3) p. 137-143, doi:10.1134/S1028335808030063.

[9]. Afonin, S.M., Solution of the wave equation for the control of an elecromagnetoelastic transduser. Doklady mathematics, 2006. 73(2), p. 307-313, doi:10.1134/\$1064562406020402.

[10]. Cady W.G., Piezoelectricity: An introduction to the theory and applications of electromechancial phenomena in crystals. New York, London: McGraw-Hill Book Company, 1946. 806 p.

[11]. Physical acoustics: Principles and methods. Vol.1. Part A. Methods and devices. Mason, W., Editor, New York: Academic Press, 1964. 515 p.

[12]. Zwillinger, D., Handbook of differential equations. Boston: Academic Press, 1989. 673 p. 
[13]. Afonin, S.M., Structural-parametric model and transfer functions of electroelastic actuator for nano- and microdisplacement. Chapter 9 in Piezoelectrics and nanomaterials: Fundamentals, developments and applications. Parinov, I.A., Editor, New York: Nova Science, 2015. p. 225-242.

[14]. Afonin, S.M., A structural-parametric model of electroelastic actuator for nano- and microdisplacement of mechatronic system. Chapter 8 in Advances in nanotechnology. Volume 19. Bartul, Z., Trenor, J., Editors, New York: Nova Science, 2017. p. 259-284.

[15]. Afonin, S.M., Nano- and micro-scale piezomotors. Russian engineering research, 2012. 32(7-8): p. 519522, doi:10.3103/S1068798X12060032.

[16]. Afonin, S.M., Generalized parametric structural model of a compound electromagnetoelastic transducer. Doklady physics, 2005. 50(2) p. 77-82, doi: 10.1134/1.1881716.

[17]. Afonin, S.M., Elastic compliances and mechanical and adjusting characteristics of composite piezoelectric transducers. Mechanics of solids, 2007. 42(1): p. 43-49, doi:10.3103/S0025654407010062.

[18]. Afonin, S.M., Stability of strain control systems of nano-and microdisplacement piezotransducers. Mechanics of solids, 2014. 49(2): p. 196-207, doi:10.3103/\$0025654414020095.

[19]. Afonin, S.M., Structural-parametric model electromagnetoelastic actuator nanodisplacement for mechatronics. International journal of physics, 2017. 5(1): p. 9-15, doi:10.12691/ijp-5-1-2.

[20]. Afonin, S.M., A block diagram of electromagnetoelastic actuator nanodisplacement for communications Systems. Transactions on Networks and Communications, 2018. 6(3): p. 1-9, doi:10.14738/tnc.63.4641.

[21]. Afonin, S.M., Electromagnetoelastic nano- and microactuators for mechatronic systems. Russian Engineering Research, 2018. 38(12): p. 938-944, doi:10.3103/S1068798X18120328.

[22]. Springer handbook of nanotechnology. Bhushan, B., Editor, Springer, Berlin, New York, 2004. 1222 p.

[23]. Encyclopedia of nanoscience and nanotechnology. 10-Volume set. Nalwa, H.S., Editor. American Scientific Publishers, Los Angeles, 2004. 